Mots. Les langages du politique

$80 \mid 2006$

La politique mise au net

\title{
Un exemple d'utilisation du courriel pour diffuser une proposition citoyenne
}

Philippe Breton

\section{OpenEdition}

Journals

Édition électronique

URL : https://journals.openedition.org/mots/534

DOI : $10.4000 /$ mots.534

ISSN : 1960-6001

Éditeur

ENS Éditions

Édition imprimée

Date de publication : 1 mars 2006

Pagination : 61-72

ISBN : 2-84788-095-X

ISSN : 0243-6450

Référence électronique

Philippe Breton, "Un exemple d'utilisation du courriel pour diffuser une proposition citoyenne », Mots. Les langages du politique [En ligne], 80 | 2006, mis en ligne le 01 mars 2008, consulté le 22 avril 2022. URL : http://journals.openedition.org/mots/534 ; DOI : https://doi.org/10.4000/mots.534 


\section{Un exemple d'utilisation du courriel pour diffuser une proposition citoyenne}

Cet article présente l'analyse d'une « expérience de communication » réalisée sur Internet en avril 2002. Il s'agissait de convaincre, via un envoi de courriels, un certain nombre de personnes - qui étaient inconnues de l'auteur du message - de participer à l'« initiative citoyenne » que ce dernier leur proposait. Le contexte de cette expérience est celui de la présence, au second tour de l'élection présidentielle de 2002, de Jean-Marie Le Pen, candidat du Front national. L'initiative proposée aux destinataires de ce message consistait à offrir une «formation » au dialogue avec des électeurs du Front national afin que ces derniers renoncent à cette option.

Sur le plan scientifique, cette expérience est intéressante à observer à plusieurs titres, notamment du point de vue de l'articulation - et de la tension entre un support de communication (Internet), un moyen de communication (l'écriture) et un genre de communication (l'argumentation). Le support et le moyen qui, ici, privilégient la communication à distance, sont-ils compatibles avec le genre, l'argumentation?

En somme, peut-on argumenter efficacement (c'est-à-dire convaincre) par courriel auprès d'un auditoire que l'on ne connait pas, là où la tradition rhétorique fait plutôt de la présence de l'orateur et de l'auditoire dans le même espace physique, celui d'une « assemblée », une condition sine qua non de l'efficacité argumentative?

\section{Deux conditions pour convaincre}

On formulera dans cet article, à propos de cette question, l'hypothèse selon laquelle au moins deux conditions doivent être impérativement remplies pour qu'un message diffusé par écrit sur Internet ait quelque chance de convaincre.

La première condition concerne le message lui-même, qui doit être rédigé de façon explicitement et soigneusement argumentative et respecter certains 
canons rhétoriques, notamment du point de vue de la distinction avec d'autres genres de communication mais aussi du point de vue de sa construction importance de l'exorde et de la formulation claire d'arguments.

La seconde concerne l'auditoire et implique le renoncement à s'adresser à un auditoire universel, au profit d'une adresse directe à une communauté de réception clairement identifiée dans le message lui-même, et à laquelle, de ce fait, des arguments spécifiques peuvent être adressés.

Il s'agit, dans le premier cas, de mobiliser toutes les ressources dont dispose l'écrit pour déployer le genre argumentatif hors de son « espace naturel », et, dans le second, d'intégrer dans le message un « accord préalable », au sens que Chaïm Perelman et Lucie Olbrechts-Tyteca (1970) donnent à cette notion.

On constatera qu'en dehors d'un aspect indéniablement pratique, il n'y a pas de spécificité d'Internet dans cette affaire, le vrai problème se situant dans la capacité à reconstituer à l'écrit les conditions orales qui sont le berceau naturel du genre argumentatif. La tension principale qu'il faut donc résoudre réside entre le genre de communication (l'argumentatif) et le moyen de communication (l'écrit), le support (Internet) ne jouant qu'un rôle secondaire.

L'analyse du message envoyé lors de l'expérience décrite ici, comme de la réception dont il a été l'objet, permet, sinon de faire la preuve - il faudrait pour cela une répétition de l'expérience -, du moins d'illustrer le fait que lorsque ces conditions sont remplies, les chances de convaincre sont meilleures.

\section{En toile de fond}

Ces deux conditions doivent être rapportées à une problématique d'ensemble qu'il est nécessaire de rappeler avant de faire l'analyse détaillée de l'expérience. Trois points peuvent être mentionnés à cette occasion, qui en constituent la toile de fond.

Le premier concerne la nécessité de distinguer les genres de communication (sur cette typologie, ainsi que sur la distinction entre genres, moyens et supports, voir Breton et Proulx, 2006c). À la suite de Jürgen Habermas, qui propose de faire le départ entre trois types d' «agir communicationnel » qui renvoient à des "réalités objectives, normatives ou subjectives» (1987, p. 132), il semble que l'on puisse distinguer trois genres principaux de la communication, qui relèvent respectivement de l'informatif, de l'argumentatif et de l'expressif.

Cette distinction, aussi bien que celle d'Habermas, s'inscrit dans la partition classique qu'avait inaugurée Aristote entre les analytiques, l'argumentatif (incluant la dialectique) et la poétique (sur les vicissitudes de l'histoire de cette partition et de la réception du genre argumentatif, voir Breton, 2006 a).

Cette partition (d'autres sont bien sûr tout à fait légitimes) présente bien des avantages, notamment du point de vue de l'analyse de la nature du con- 
trat implicite passé entre l'émetteur d'un message et son récepteur. Elle permet aussi de mieux comprendre que l'efficacité d'un message argumentatif est aussi liée à sa capacité à maintenir et à exhiber une frontière étanche avec le domaine de l'informatif et de l'expressif. Sa construction mobilise donc une compétence particulière, qui consiste à savoir identifier et séparer les genres au sein d'un même message.

Le fait que le message soit écrit semble renforcer considérablement cette nécessité d'une construction rigoureuse, compte tenu de la faible capacité de rétroaction mutuelle dont disposent à la fois l'auteur du message et son récepteur. C'est à ce point précis que la tension entre le moyen et le support entre en ligne de compte. Le basculement d'une partie de l'écrit sur Internet, notamment grâce au courrier électronique, n'est pas forcément synonyme d'un maintien de l'attention vis-à-vis des règles de l'écrit.

Un certain relâchement, rationalisé le plus souvent comme positif, voire même associé à la modernité de l'outil, peut conduire, s’il était attesté, à une moindre compétence notamment à reconstituer les conditions idéales pour garantir l'efficacité argumentative du message.

Une autre distinction doit être mobilisée dans cette analyse, celle qui sépare la communication directe, en présence, et la communication indirecte, médiatisée. C'est l'objet du deuxième point. L'irruption des nouvelles technologies dans le domaine de la communication, l'offre tous azimuts de médias qui permettent le transport des messages - Robert Escarpit (1976) n'avait-il pas défini la communication comme un "cas particulier du transport»? - ont largement augmenté la part de la communication médiatisée dans l'ensemble des échanges communicationnels.

Cela avait déjà été le cas avec l'irruption de l'écriture, notamment sous sa forme alphabétique, dans le monde antique et notamment en Grèce. On sait depuis Socrate - grâce à la mise en scène de son point de vue dans le Phèdre de Platon - que le recours à la communication médiatisée ne va pas de soi, là où le dialogue direct a d'évidentes vertus. Comme le rappellent C. Perelman et L. OlbrechtsTyteca, « dans un dialogue, celui qui argumente peut s'assurer, au fur et à mesure qu'il avance, de l'adhésion de son interlocuteur aux chaînons du raisonnement. C'est à cette technique que fait allusion Socrate » (édition de 1977, p. 45).

Plane donc toujours la suspicion d'une ambivalence possible du progrès en matière de communication, qui associe inévitablement un éventuel surcroit de puissance avec une possible perte de sens.

La question de la perte est d'ailleurs au centre de la problématique des nouvelles technologies de communication, d'abord sur le plan du signal, mais aussi sur celui du sens. Ces technologies, dont le berceau historique est la «théorie mathématique de l'information » de Claude Shannon, dans les années quarante, sont d'ailleurs nées d'une recherche d'efficacité et d'optimisation dans le domaine du transport du signal comme porteur de sens. 
Il est donc important de croiser une réflexion en termes de genre et en termes de perte. Autant l'information se trouve, si l'on peut dire, à l'aise dans la communication médiatisée, du fait du processus d'objectivation dont elle est précisément le produit, autant cette question doit être posée dans le cas de l'opinion et des arguments qui l'accompagnent nécessairement.

Peut-on convaincre à distance? La perte, en ligne, n'est-elle pas trop importante? L'espace physique, celui de la coprésence, dans une assemblée où chacun se fait face (Détienne, 2003), pour échanger à l'oral, n'est-il pas le terreau privilégié d'un échange argumentatif qui, par ailleurs, se défie de l'écrit pour privilégier l'adresse directe à l'oral - ou en langue des signes, pour les personnes sourdes (Delaporte, 2002)? L'argumentation, comme acte de parole, n'estelle pas soudée à la présence?

Cette question appelle une troisième remarque, qui concerne l'auditoire. L'activation, sur Internet, de communications entre des personnes qui ne se connaissent pas, et qui ne peuvent donc pas anticiper leur réactions mutuelles, peut-elle provoquer un effet de communauté, bref, générer la formation d'un auditoire particulier? En somme, l'outil fait-il lien?

La croyance quasi religieuse qui est au cœur du discours d'accompagnement d'Internet (Breton, 2000) veut que le support de communication «sanctifie » ce qu'il transporte et qu'ainsi, en quelque sorte mécaniquement, un meilleur transport soit synonyme de meilleure qualité de ce qui est transporté. Un signal efficient serait la garantie d'un « meilleur sens». L'usage de la technique ferait communauté, car il favoriserait une meilleure communication.

L'hypothèse inverse est pourtant plus réaliste. Les chances de succès d'un message - notamment dans le genre argumentatif - dépendent peut-être du fait qu'il s'adresse - et qu'il est approprié - à une communauté de réception préexistante, constituée indépendamment du support de communication (problème que ne rencontre pas le message informatif, qui est d'emblée conçu pour un auditoire universel, même si l'universalité est toujours relative dans ce caslà). C'est uniquement à cette condition que l'émetteur peut construire une argumentation qui s'appuie sur un accord préalable, et espérer établir un lien avec l'auditoire dont il espère modifier l'opinion ou entrainer l'adhésion.

\section{Convaincre à distance}

L'«expérience de communication » qui sert ici en quelque sorte de support à la question posée - peut-on, et à quelles conditions, argumenter avec succès en utilisant le courriel sur Internet - consistait donc à proposer, à des personnes susceptibles de s'y intéresser, une "formation citoyenne » à l'argumentation, dans le but d' " apprendre » à convaincre des électeurs du Front national de renoncer à leur option électorale et à leur choix politique, cela dans le con- 
texte « traumatique » de la période qui a suivi immédiatement l'élection présidentielle de $2002^{1}$.

À partir de là, la question était de savoir comment faire connaitre cette « proposition citoyenne», comment convaincre les destinataires du message de se joindre à cette initiative et comment assurer techniquement au message une présentation qui garantisse, en termes d'ethos, l'intégrité de l'auteur du message aux yeux de ses lecteurs (en l'occurrence, une présentation qui convainque qu'il ne s'agissait ni d'une proposition fantaisiste, ni d'une secte dissimulée, ni d'un groupe politique cherchant à recruter « par la bande », ni enfin d'une opération de désinformation montée par l'extrême droite ou d'autres).

Plusieurs options étaient possibles: le bouche à oreille dans un environnement amical et professionnel (mais cela aurait pris beaucoup de temps, sans garantie de succès), l'entrée en contact avec un média local, qui aurait fait un article à ce sujet (mais il y aurait eu, dans ce cas, le poids d'une médiation), ou le recours à des associations ou à des organisations politiques (mais le message, là encore, aurait été « contaminé » par cette médiation).

L'option d'un message envoyé par écrit à un grand nombre de personnes s'est donc imposée. Internet, grâce à la fonction du courriel - qui est une sorte de service postal amélioré -, permettrait alors de pratiquer une diffusion sur le mode du broadcasting (ce mode de diffusion est ainsi nommé initialement en référence métaphorique au geste du semeur, qui « sème à tout vent »).

Le message a donc été envoyé à l'adresse électronique d'un millier de personnes habitant la ville ou la région de Strasbourg, soit des individus isolés, soit des membres ou des responsables d'associations, d'organisations et de partis. Ce carnet avait été constitué dans un tout autre contexte et un certain nombre - mais pas tous - de ceux qui y figuraient étaient supposés avoir une sensibilité « citoyenne».

\section{Construire le message}

Il s'agissait donc de rédiger un message suffisamment convaincant pour déclencher l'intérêt et l'adhésion. De plus, proposant une formation à l'argumentation, le message devait en lui-même témoigner de la capacité argumentative de son auteur. Voici, intégralement, le texte initial:

1. L'auteur de l'article est à l'initiative de cette expérience, qu'il a réalisée seul, en dehors de toute institution, si ce n'est la référence à son institution d'appartenance, le CNRS. Parler ici d' « expérience de communication » ne signifie pas que le but de cette initiative était de faire une expérience en vue de son analyse. Cette initiative a été prise dans un cadre «citoyen » et a rempli indépendamment toute sa fonction dans ce cadre (les ateliers proposés ont bien fonctionné). Elle n'a été analysée qu'a posteriori comme une expérience, à la demande de la revue qui publie cet article. En somme, le citoyen a agi et le chercheur a analysé après coup l'action, en l'objectivant du mieux qu'il était possible. On a tenté de limiter ici les conséquences du fait que le citoyen 
From: Philippe Breton phbreton@club-internet.fr

Reply-To: phbreton@club-internet.fr

To: undisclosed-recipients

Subject: Convaincre

Date: Thu, 25 Apr 2002 15: 09: 02 + 0200

Madame, Monsieur

J'ai pensé que, dans la situation actuelle, cette initiative pouvait vous intéresser, ou l'un de vos proches. Merci d'y consacrer un peu d'attention.

Cordialement

Philippe Breton

Chercheur au CNRS (sciences de la communication)

Pour défendre la démocratie: les ateliers civiques d'argumentation

Vous vous demandez quoi faire modestement et concrètement devant la grave crise que connait la démocratie dans notre pays?

Vous pensez que tous les électeurs de l'extrême droite ne sont pas forcément des fascistes mais bien souvent des personnes qui ont cru, parfois faute de mieux, pouvoir se faire entendre de cette façon-là.

Vous avez, dans votre entourage proche, des électeurs de l'extrême droite, parents, amis, collègues, voisins, connaissances, qu'il serait peut-être possible, malgré les apparences, de convaincre de renoncer à cette voie, notamment dans les prochaines consultations électorales.

Vous ne savez pas comment faire pour prendre la parole et argumenter dans cette situation difficile, mais vous auriez envie de le faire et d'apporter de cette façon une contribution personnelle concrète à la démocratie.

Je vous propose une initiative originale dont l'idée est née de la situation actuelle: échanger, débattre et suivre une «formation » dans un « atelier civique d'argumentation ». L'objectif spécifique est d'apprendre à s'adresser à des personnes qui sont tentées de sortir de la démocratie, pour les convaincre de faire machine arrière et de se faire entendre autrement.

Ces séances, organisées en après-midi et en soirée, en fonction des possibilités, se dérouleraient sur le modèle des formations à l'argumentation, à la prise de parole, à la communication, par exemple en trois étapes, de la façon suivante:

1 - Échanges d'expériences vécues, à qui parle-t-on, les obstacles à la parole, la question de la violence verbale.

2 - Enjeux et techniques de l'argumentation: comment s'y prendre pour établir le dialogue, pour convaincre? Comment se construit une argumentation?

3 - Échanges, réflexions et apports sur le thème: quels arguments concrets peut-on utiliser dans cette situation?

Ces « ateliers civiques d'argumentation », conçus comme de véritables lieux de formation, sont libres, ouverts et gratuits (sauf partage des frais de location des sal-

et le chercheur sont une seule et même personne mais aussi du fait qu'il peut y avoir des dissensus entre ces deux approches, qui ont chacune leurs méthodes et leurs finalités propres. Que l'initiative soit elle-même en lien avec le champ de l'argumentation est sans rapport avec l'analyse de l'acte argumentatif qui consiste à convaincre de participer à cette initiative. 
les), indépendants des engagements politiques, et tournés vers un seul objectif: défendre concrètement la démocratie avec ce qui en constitue le cœur: la parole. Si vous êtes intéressés ou souhaitez de plus amples informations sur la mise en place de ces ateliers, vous pouvez contacter Philippe Breton au 06 xx xx xx ou par courrier électronique: phbreton@club-internet.fr

\section{De nombreuses réponses}

Comme bouteille à la mer, on ne pouvait pas faire mieux ! De nombreuses personnes ont pourtant répondu dans les jours qui ont suivi, essentiellement par mail $^{2}$, quelques-unes par téléphone.

Autre conséquence de cet envoi: le quotidien régional, qui a eu vent de l'initiative, a cru bon de relayer l'information par un petit encart. À titre privé, un journaliste s'inscrira d'ailleurs à l'un des ateliers. Plusieurs appels téléphoniques nous sont également parvenus, émanant de personnes se présentant comme « électeurs du FN » et souhaitant, in fine, dialoguer - in fine, car le contact, au début, a été plus... rude (voir à ce sujet, Breton, 2006b).

Devant le nombre relativement important de réponses, un second message a été rapidement envoyé à l'ensemble des répondants.

Vous avez été nombreux à manifester votre intérêt pour l'initiative des « ateliers civiques d'argumentation ». Voilà ce que je vous propose, plus précisément. Pour commencer, je me présente: je suis chercheur au CNRS et j'anime des formations à l'argumentation, à destination de différents milieux professionnels (enseignants, cadres, magistrats). C'est en me demandant ce que je pouvais personnellement apporter dans le cadre de la situation actuelle que j'ai eu l'idée de partager mon « savoirfaire » dans ce domaine. Je viens d'être conforté dans ce projet par les résultats d'une enquête récente qui indique que «seul» le tiers des électeurs du FN avait des idées d'extrême droite et que les autres agissaient ainsi pour d'autres raisons.

Je propose donc d'organiser une journée, sur le modèle d'une journée de formation "classique», qui se déroulerait en gros de la façon suivante: présentation mutuelle - motivations des uns et des autres - échanges sur des situations vécues (rencontres avec des personnes soutenant l'extrême droite) - qu'est ce qu'argumenter? - les enjeux de l'argumentation - le rapport à la violence et à la civilité la différence argumentation/manipulation - comment préparer une argumentation - les obstacles à la parole - l'entrée dans le dialogue - comment tirer de la connaissance et de l'écoute de l'autre les éléments d'une argumentation - les grandes familles d'arguments - quels arguments spécifiques utiliser dans cette situation. J'animerai évidement moi-même cette journée. Le premier groupe ne devrait pas dépasser quinze personnes, afin que nous puissions travailler sérieusement. Il ne s'agit pas d'une formation «universitaire» et tout sera fait pour que chacun, quel

2. Voici un petit comptage des réponses dans les premiers jours : 25 avril : 11 messages ; 26 avril : $8 ; 27$ avril : $2 ; 28$ avril : 6, 29 avril : $1 ; 30$ avril : 7, etc. L'immense majorité d'entre eux témoignaient d'un intérêt positif pour l'initiative. 
que soit son point de départ, s'y sente à l'aise. L'important est notre motivation commune. [...]

Il est toutefois important que vous m'indiquiez si vous souhaitez participer à cette journée, pour des questions évidentes d'organisation.

Voilà, si vous souhaitez d'autres précisions ou en parler directement, vous pouvez me joindre à mon numéro privé: $x x x x x x x x$, sinon, envoyez-moi un e-mail pour confirmer, si c'est le cas, votre présence.

Très cordialement

Philippe Breton

Puis un nouveau message a été envoyé à l'ensemble des adresses de la liste initiale, libellé ainsi:

Cher(e) ami(e)

Suite à l'initiative dont je vous avais tenu informé dans un précédent mail, je vous signale qu'un premier « atelier » aura lieu le samedi 11 mai prochain, de $9 \mathrm{~h} 30$ à $17 \mathrm{~h}$. Je viens d'être conforté dans ce projet par les résultats d'une enquête récente qui indique que «seul» le tiers des électeurs du FN avait des idées d'extrême droite et que les autres agissaient ainsi pour d'autres raisons. Il s'agira de nous confronter ensemble aux problèmes posés (et aux «techniques » d'argumentation correspondantes) par le dialogue avec ces électeurs du Front national. Inutile de rappeler que cette question risque d'être encore d'actualité pour un certain temps.

Il reste deux ou trois places pour cette première séance. Si vous êtes intéressés, n’hésitez pas à prendre contact par mail.

Cordialement

Philippe Breton

\section{Les raisons d'un succès}

La suite de cette expérience a été concluante sur plusieurs plans. Différents ateliers (de dix à douze participants) se sont déroulés, concernant en tout une petite centaine de personnes. Puis les médias s'en sont fait l'écho ${ }^{3}$. Il n'est plus possible, à partir de ce moment-là, d'évaluer le strict effet du message électronique initial. L'évaluation de l'expérience s'arrête donc à ce stade.

Au départ, il y a un message, écrit, sur Internet, support de communication le plus indirect qui soit, adressé par un inconnu à des inconnus; à l'arrivée, plus d'une centaine de personnes convaincues et acceptant de suivre ensemble un atelier pendant une journée entière, cela pour être les relais d'une initiative citoyenne visant à s'adresser individuellement à des électeurs du Front national afin de les dissuader de continuer à l'être.

3. L'un des ateliers (avec l'accord des participants) s'est déroulé en présence d'un journaliste du quotidien Libération, qui a réalisé une double page de reportage sur le sujet. Un autre atelier s'est tenu en présence d'une équipe de France Inter, qui a diffusé, pratiquement sans commen- 
On peut considérer, ici, qu'il s'agit d'un succès. Ce résultat dépasse en tout cas nos prévisions. Comment expliquer la réussite de l'initiative? Examinons les deux conditions évoquées plus haut et voyons si elles sont remplies.

\section{Un texte réellement argumentatif}

La première condition implique que le message soit rédigé sur un mode argumentatif (au sens qu'Aristote donnait à la rhétorique comme « art de convaincre »). Cela peut apparaitre comme une évidence. Pourtant, l'examen de nombreux textes, ou prises de parole orales, qui visent explicitement à convaincre un auditoire, montre que leur construction ne respecte guère les normes que l'on peut attendre d'un tel genre.

Une autre expérience, réalisée dans un contexte oral et dans des conditions rigoureuses de reproductibilité et de réitération, souligne de sérieux problèmes de compétences pour mettre dans une forme argumentative un message visant à convaincre (Breton, 2005b). Les difficultés dans la maitrise de l'écrit, ainsi que les illusions rationalisantes dont Internet est entouré (voir plus haut), s'ajoutent à cette incompétence initiale.

On ne jugera pas de la qualité argumentative des messages qui sont analysés ici. On retiendra simplement qu'une très grande attention a été portée à leur rédaction, à partir des principes suivants:

- ce n'est pas parce que le message est transmis par courriel qu'il ne doit pas être rédigé comme une lettre et bénéficier ainsi de toute l'expérience acquise dans le domaine épistolaire (sur laquelle certains voudraient tirer un trait sans se rendre compte de l'immense perte d'efficacité que cela représenterait);

- ce n'est pas parce que le message est écrit qu'il ne doit pas s'adresser à l'auditoire, un peu comme on le ferait à l'oral. L'écrit, ici, n'appartient pas au genre expressif et doit donc renoncer au style « littéraire »; il est au service d'une argumentation, donc d'une opinion que l'on propose de partager;

- le texte part d'une conviction revendiquée comme telle et ne se cache pas derrière des frontières poreuses, derrière un genre informatif et démonstratif qui relèverait ici, de façon inopportune, de l'évidence (voir les développements de Perelman et Olbrechts-Tyteca sur ce thème, 1970);

- le texte respecte les canons de la construction classique du genre argumentatif en jouant à la fois sur l'ethos, le pathos et le logos et surtout, en respectant l'architecture de ce genre: exorde, exposé contextualisé de l'opinion, arguments, péroraison.

taire, dans un programme réalisé par la journaliste Zoe Varier, deux heures entières des débats qui s’y étaient tenus. La demande insistante d'une équipe de l'émission de télévision «Striptease », ainsi que celle d'un producteur privé, ont été par contre repoussées. 


\section{La création d'un auditoire}

La deuxième condition qui assure une possible efficacité argumentative du message implique, nous l'avons dit, le renoncement à une adresse universelle, mais aussi son corollaire, "la connaissance de ceux que l'on se propose de gagner (comme) condition préalable de toute argumentation efficace » (Perelman et Olbrechts-Tyteca, 1970, p. 26).

De fait, le message, dans son énoncé, découpe son auditoire presque sur mesure. Il annonce explicitement s'adresser à une catégorie très particulière de personnes. En résumé, ce sont celles qui:

- sont en désaccord avec les positions de l'extrême droite;

- souhaitent un changement et une diminution de l'influence de ces positions;

- sont attachées à la démocratie dans la pratique;

- sont au contact physique de personnes qui soutiennent par leur vote les positions de l'extrême droite;

- ne pensent pas que tous les électeurs de l'extrême droite sont forcément d'extrême droite eux-mêmes;

- ne savent pas comment s'y prendre pour, concrètement, leur parler et les faire changer d'avis.

On voit bien qu'il ne s'agit pas d'un auditoire « universel», mais bien du segment des personnes effectivement susceptibles d'être intéressées par l'initiative proposée car, toujours selon Perelman et Olbrechts-Tyteca:

Pour qu'il y ait argumentation, il faut que, à un moment donné, une communauté effective des esprits se réalise. Il faut que l'on soit d'accord, tout d'abord et en principe, sur la formation de cette communauté intellectuelle et, ensuite, sur le fait de débattre ensemble d'une question déterminée: or, cela ne va nullement de soi. (1970, p. 18)

Nous sommes donc bien dans la stratégie de l'accord préalable qui semble être au cœur de l'argumentation (Breton, 2005a). Celle-ci implique, comme l'avaient vu Perelman et Olbrechts-Tyteca, que l'on s'assure pas à pas de cet accord, c'està-dire qu'on en rappelle les termes, dans l'argumentation elle-même, à l'auditoire. Le message doit donc proposer à ses lecteurs une image de ce qu'ils sont et leur rappeler ce à quoi ils sont attachés, ce sur quoi l'orateur prendra appui.

Le problème ici était de savoir si cet auditoire, construit par l'auteur du message, correspondait à un auditoire réel. En fait, c'est sur ce point que tout le risque de l'aventure reposait. De nombreuses actions militantes de l'époque tablaient sur l'hypothèse que les électeurs de l'extrême droite représentaient une réelle menace pour la démocratie, qu'ils savaient ce qu'ils faisaient, étaient 
informés des attendus et des conséquences de leurs votes et qu'il fallait donc les prendre au pied de la lettre.

Dans cet esprit, seul le combat frontal permettait d'isoler et de circonscrire cette maladie du corps social qu'ils incarnaient et qui menaçait de contaminer l'ensemble, tel un cancer politique. Certains se voyaient comme de nouveaux résistants prêts à prendre courageusement les armes, des armes idéologiques dans un premier temps. Ceux qui proposaient le dialogue avec les électeurs du Front national passaient pour des « collabos ».

Dans l'exaltation des comités antifascistes influencés par l'extrême gauche, certains fantasmes allaient dans le sens de l'enfermement, à titre préventif, des électeurs les plus actifs du Front national... Comme le rappelle Jacques Le Bohec, « dans ces conditions, rencontrer des électeurs pour en savoir plus et mieux paraît inutile et suspect. Les rencontrer c'est se salir. Les comprendre c'est se compromettre » (2005, p. 70).

Ce n'était évidemment pas à de tels auditoires que s'adressait le message, mais plutôt à ceux qui, d'une part, ne voyaient pas les électeurs du Front national comme des êtres politiques rationnels adhérant en toute conscience aux thèses idéologiques de ce parti - lequel n'est d'ailleurs pas du tout monolithique de ce point de vue -, et qui, d'autre part, avaient dans leur entourage proche des personnes ayant voté pour cette tendance sans pouvoir être, à l'évidence, considérées comme «fascistes » (rappelons que l'expérience se déroule en Alsace, région qui a donné plus de $25 \%$ de ses voix au Front national à la présidentielle de 2002, ce qui signifie que beaucoup de personnes côtoient, théoriquement, dans leur entourage proche, famille ou lieu de travail, de tels électeurs).

L'impératif d'efficacité du message impliquait donc qu'il construise effectivement un auditoire qui ne se percevait pas forcément comme tel auparavant. Il s’appuie de ce fait sur une communauté virtuellement préexistante et il contribue à la faire exister comme telle à ses propres yeux.

De ce point de vue, le pari a été tenu et cet auditoire, comme l'a montré la suite des opérations, existait bien. Il était même composé de nombreuses personnes qui, souvent, se croyaient seules dans cette situation et se taisaient face à la doxa, celle de l' " antifascisme » militant qui dénonçait à pleins poumons le retour imminent du nazisme prétendument incarné par ces $25 \%$ d'électeurs.

Le simple fait de répondre au message initial leur a permis une prise de conscience collective. Leurvolonté d'agir, jusque-là paralysée, a pu se déployer à travers la proposition qui leur était faite. Le message initial a tout à la fois construit et trouvé son auditoire.

L'efficacité argumentative, dans ce sens, est liée au fait que le message ajoute un maillon décisif à une chaine qui relie l'auditoire tout en le renforçant comme tel. Que l'argumentation restaure le lien social dans un domaine où il est précisément défaillant - nous découvrirons d'ailleurs que de nombreux électeurs du Front national font ce choix précisément parce qu'ils se sentent exclus, et que 
le seul fait de rétablir le dialogue avec eux les éloigne de leur choix initial-, que l'argumentation permette de renouer une chaine rompue correspond bien, au fond, à la finalité ultime de l'acte argumentatif dans une société démocratique, qui est de créer et d'entretenir un lien social respectueux, tout simplement, de l'humain.

Internet, on le voit bien ici, n'a joué finalement que le rôle secondaire d'un média de mise en contact, intervenant dans une communauté de problème qui lui est préexistante. En somme, ce n'est pas l'outil qui a fait communauté, mais la communauté qui a utilisé l'outil.

\section{Références}

ARISTOTE, Rhétorique, texte établi et traduit par Médéric Dufour, 1967, t. 1, 2 et 3, Paris, Les Belles Lettres.

BRETON Ph., 2000, Le culte de l'Internet, Paris, La Découverte.

- 2005a, L'argumentation dans la communication, Paris, La Découverte (Repères).

- 2005b, «Présentation et analyse d'un cadre expérimental d'apprentissage des compétences démocratiques", Cahiers pédagogiques, nº 433, mai, La démocratie à l'école, p. 28-29.

-2006 a, «Between science and rhetoric: a recurrent debate on the role of communication and creativity in the definition of knowledge », A. Sales et M. Fournier éd., Knowledge, Communication and Creativity, Londres, Sage Publications.

- 2006b, Argumenter en situation difficile, Paris, Pocket.

- et PROULX S., 2006c, L'explosion de la communication, Paris, La Découverte.

DELAPORTE Y., 2002, Les sourds, c'est comme ça, Paris, MSH.

DETIENNE M. éd., 2003, Qui veut prendre la parole?, Paris, Le Seuil (Le genre humain).

ESCARPIT R., 1976, Théorie générale de l'information et de la communication, Paris, Hachette Université.

HABERMAS J., 1987, Théorie de l'agir communicationnel, Paris, Fayard.

LE BOHEC J., 2005, Sociologie du phénomène Le Pen, Paris, La Découverte (Repères).

PERELMAN Ch. et OLBRECHTS-TYTECA L., 1970 [1958], Traité de l'argumentation. La nou-

velle rhétorique, Bruxelles, Éditions de l'Université de Bruxelles (première édition: Paris, Presses universitaires de France).

PLATON, Phèdre, trad., introduction et notes par L. Brisson, 1997, Paris, GF-Flammarion. 